\title{
An Indoor Electromagnetic Testing Antenna Design with Better Standing Wave Ratio and Gain
}

\author{
H. Lv, X. S. Xia, Y. L. Yu, Z. X. Hua \\ Anhui Jianzhu University \\ Hefei, Anhui, China
}

\begin{abstract}
Electromagnetic radiation harm human health serious, indoor household appliances electromagnetic radiation spectrum, not only more, but also broad. In order to effectively detect the indoor electromagnetic wave, the paper puts forward an antenna electromagnetic detection, using slotted and add a high impedance method such as micro-strip to broadening the broadband antenna. Through the simulate analysis of HFSS, The results show that-10dB return loss bandwidth covers a frequency range between $0.14 \mathrm{GHz}$ and6. $3 \mathrm{GHz}$. In other words, the relative bandwidth is $191.3 \%$.
\end{abstract}

Keywords-indoor; electromagnetism detection; antenna design

\section{INTRODUCTION}

Electromagnetic radiation hazards to human has been confirmed in academia[1-2], the State Environmental Protection Administrationin2000, the first completed national survey of pollution sources of electromagnetic radiation[3], the findings show that electromagnetic radiation such as radio communications and radar has been issued for a pollution . With the development of technology, the popularity of home appliances to accelerate, while bringing magnetic pollution, these electromagnetic waves flooded space, colorless, odorless, invisible, can penetrate any substance including human to human pollution. According to statistics, the national mobile phone users over one billion[4], indoor growing problem of electromagnetic pollution, so the indoor electromagnetic radiation detection imminent.

Currently, the majority of indoor electromagnetic detection antenna band is narrow, and just for indoor some appliances electromagnetic radiation band. Such as mobile phone antenna effective detection range of electromagnetic radiationis0. $8 \sim 1$. 5 GHzand1. $7 \sim 2.2 \mathrm{GHz}$. However, a wide variety of indoor pollution electromagnetic waves, The main radiation sources: TV:(Radiation Frequency:16 $223 \mathrm{MHz}, 471 \sim 566 \mathrm{MHz}, 607$ $\sim 958 \mathrm{MHz}$ three bands), mobile phones(radiation frequency: 900/1800MHz), microwave oven(Radiation Frequency:2. $0 \sim 3$. $5 \mathrm{GHz}$ ), washing machines, air conditioners, refrigerators, kettles and rice cookers(radiation frequency:2. $1 \sim 3.0 \mathrm{GHz}$ ), 3Gwireless network device control(5. 1 5. $8 \mathrm{GHz})$, and so on. Be seen, indoor appliances electromagnetic radiation band focused on $0.168 \sim 5.85 \mathrm{GHz}$. To be able to effectively detect the band electromagnetic indoor pollution, detection antenna should have good VSWR, approximation of omni-directional, high gain. Response to these problems, propose to achieve a form of indoor antenna detection, by HFSS simulation results show that the antenna has a good performance.

\section{ANTENNA DESIGN}

\section{A. Detection Antenna Patch Design}

UWB planar monopole antenna size is mainly determined by the low frequency antenna [5-6], for irregularly shaped radiation patch, Its planar monopole antenna VSWR ratio reaches two points corresponding to low frequency can be estimated with a cylinder approximation, Cut along a generatrix to the cylinder to obtain a rectangular, in Figure 1. Therefore, the lowest frequencies of the antenna can be written as:

$$
F_{L}=\frac{c}{\lambda}=\frac{72}{L+r+g}
$$

where c: velocity of electromagnetic waves, $\lambda$ : wavelength corresponding to the point frequency, L: width of the patch, $r$ : radius of the cylinder, g: the gap between the patch and the ground plane; units of $\mathrm{GHz}, \mathrm{L}, \mathrm{r}$ is $\mathrm{mm}$. (1. 1)according to the formula to calculate the initial size of the antennais $215 \mathrm{~mm} \times$ $111 \mathrm{~mm}$.

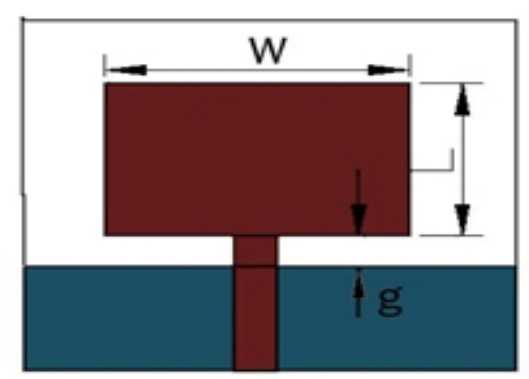

FIGURE I. FLAT RECTANGULAR RADIATING PATCH MONOPOLE ANTENNA

\section{B. Detecting Antenna Micro-strip Line Design}

Due to antenna substrate thickness is $h$, the dielectric constant of the dielectric substrate is $\varepsilon r$, the width of the radiation patch $\mathrm{w}$. Let $Z_{c}$ be the characteristic impedance of the antenna wire micro-strip line, Can be calculated by the following formula micro-strip line width $2.2 \mathrm{~mm}$.

$$
\begin{aligned}
& A=\frac{Z_{c}}{60} 0.5 \sqrt{\frac{\varepsilon_{r}+1}{2}}+\frac{\varepsilon_{r}+1}{\varepsilon_{r}-1}\left(0.23+\frac{0.11}{\varepsilon_{r}}\right) \\
& B=\frac{377 \pi}{2 Z_{C} \sqrt{\varepsilon_{r}}}
\end{aligned}
$$

For $\mathrm{A}<1.52$, are: 


$$
\frac{w_{1}}{h}=\frac{8 \exp (A)}{\exp (2 A)-2}
$$

For $\geq 1.52$, are:

$$
\frac{w}{h}=\frac{2}{\pi}\left\{B-1-\ln (2 B-1)+\frac{\varepsilon r-1}{2 \varepsilon r}[\ln (B-1)+0.39]-\frac{0.61}{\varepsilon r}\right\}
$$

\section{Detection Antenna Structure}

Through theoretical analysis, The antenna is formed on the relative dielectric constant of 2.55 , and $0.8 \mathrm{~mm}$ thickness of the dielectric substrate of polytetrafluoroethylene. Positive dielectric substrate for antenna radiation patch, patch combination of an inverted trapezoidal and formed a semicircle, and the radiation patch opened a hole[7];

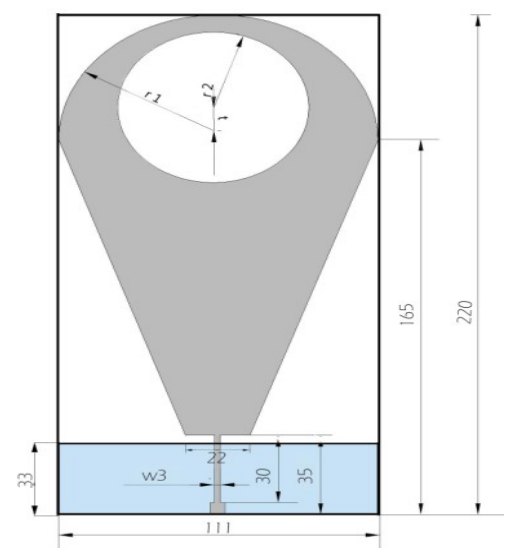

FIGURE II. THE ANTENNA STRUCTURE (UNIT MM)

\section{The Simulation Results ANALysis}

\section{A. Effect of the Band Width of the Antenna Structure}

1) Opening the Radius of Influence of Bandwidth

By HFSS simulation, optimization of the performance of the antenna, found not only to increase Bandwidth by changing the patch antenna length, width and other parameters. The study found that the shape of the metal patch antenna impedance band width impact of variable [8-9], Open a hole in the metal sheet[10], Here fixed $t=0 \mathrm{~mm}(\mathrm{t}$ is the distance between $\mathrm{r} 1 \mathrm{r} 2$ with center), Increasesr2, Figure 3 shows the return lossS11 <-10 $\mathrm{dB}$ impedance band width narrowed accordingly. The reason is that when the metal patch area is reduced, the output of the matching impedance changes, resulting in a variation of the bandwidth.

\section{2) Hole Location Impact Bandwidth}

When the fixedr2of $30 \mathrm{~mm}$, while gradually increasing $\mathrm{t}$;found from Figure 4, the low-band return loss of the antenna increases, the position of theres on ant frequency shift of the resonant intensity increases. The above combination of several factors, select $\mathrm{t}=0 \mathrm{~mm}, \mathrm{r} 2=30 \mathrm{~mm}$ conduct further research.

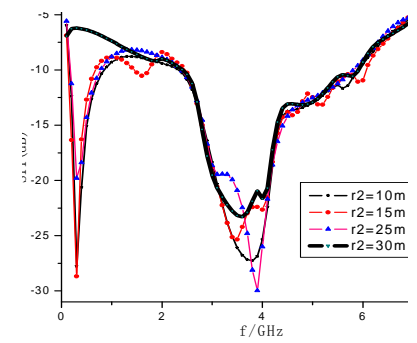

FIGURE III. S11 CHANGE WITH R2

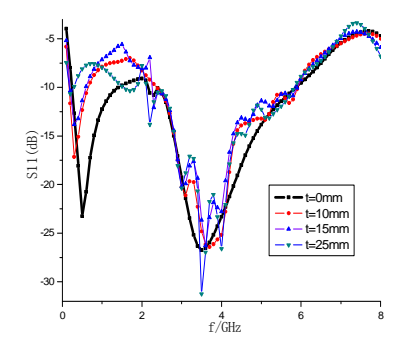

FIGURE IV. S11 CHANGE WITH T

3) Increase the Band Width of the Impact of High Impedance Micro-strip Line

When observing the input impedance of the antenna, found at relatively high frequency, The real part of the antenna in the vicinity of $50 \Omega$ fluctuations, the imaginary part of the antenna in the vicinity of $0 \Omega$ fluctuations ; At a relatively low frequency, the input impedance real part of the antenna in the vicinity of $10 \Omega$, and the relatively large negative value of the imaginary part. Described in the low frequency range, the antenna input resistance of the weak, capacitive relatively strong.

According to the principle of impedance changes[11]. Between the micro-strip feed with radiation patch increases a high impedance micro-strip, the low impedance of the micro-strip line minor change maybe equivalent to two series-connected inductor and a shunt capacitor, which can offset the capacitive input impedance of the antenna, so that the input impedance of the antenna to obtain allow end 50 is close to pure resistance. As can be seen from Figure 5, with the size of $\mathrm{Wm}$ changes, The impedance bandwidth of the antenna presents a significant change, when $\mathrm{Wm}$ is $2.2 \mathrm{~mm}$ the frequency moves to a high frequency antenna. See the bandwidth of the antenna increases, the low frequency return loss is significantly reduced whenWm1. $8 \mathrm{~mm}$ and 1.5 $\mathrm{mm}$;when $\mathrm{Wm}$ is $1.5 \mathrm{~mm}$ the impedance of the antenna bandwidth of the best. Figure 6 to come out of the detection antenna VSWR chart, it can be seen From Figure, The antenna has a very good VSWR; between $0.14 \sim 6$. 3GHz VSWR less than 2, its visible electromagnetic radiation antenna is relatively small. 


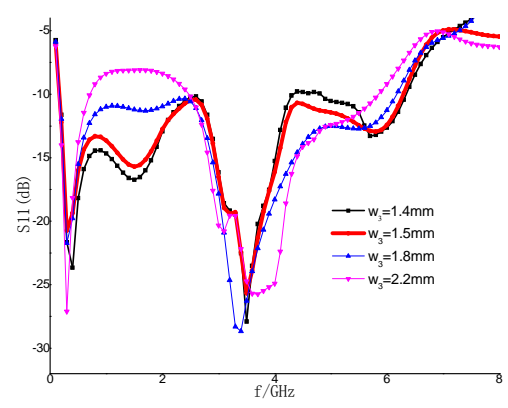

FIGURE V. S11 RETURN LOSS CHANGE WITH WM

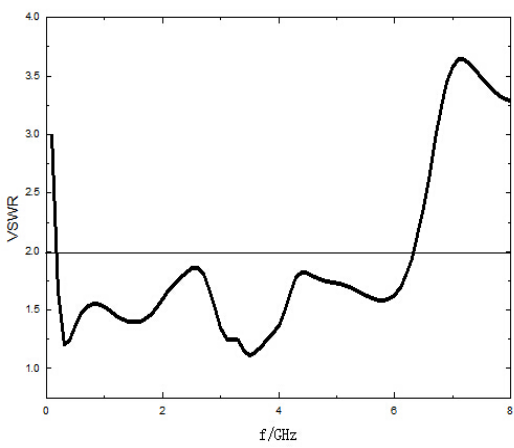

FIGURE VI. VSWR

\section{B. Directional Analysis}

Figure 7 shows the1. $8 \mathrm{GHz}, 3.5 \mathrm{GHz}, 4 \mathrm{GHz}$ frequency antenna on the H-plane radiation pattern normalized. Due to frequency of high frequency radiation affected the higher harmonic radiation, the radiation pattern in the high-frequency variation, but the entire operating band of the antenna has an approximately omnidirectional and symmetry. Figure8 showsthe1. $8 \mathrm{GHz}, 3.5 \mathrm{GHz}, 4 \mathrm{GHz}$ frequency antenna on the E-plane radiation pattern normalized, the dipole radiation field is similar to the radiation field, showing a "8" shape.

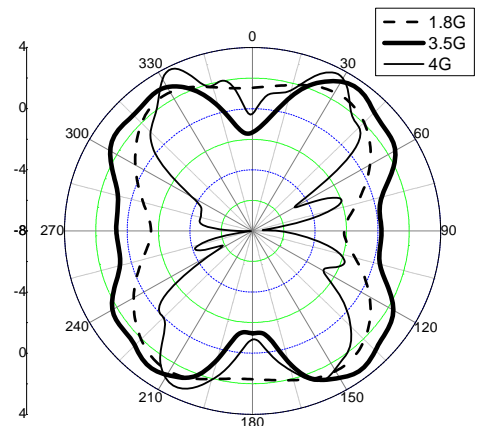

FIGURE VII. H-PLANE NORMALIZED ANTENNA PATTERN

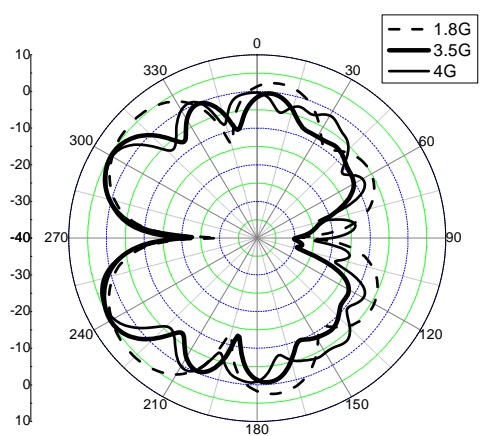

FIGURE VIII. ANTENNA E-PLANE NORMALIZED PATTERN

Through simulation, we can see that paragraph indoor antenna with good detection SWR, approximate omnidirectional, good gain. Radiation large indoor electrical products include: cooker, vacuum cleaners, microwave ovens, cell phones, etc. ; cooker, microwave frequency electromagnetic radiation is about 2. $5 \mathrm{GHz}$, Now the smart phone electromagnetic radiation frequency of about1. $8 \mathrm{GHz}$; The antenna in the $1.8 \mathrm{GHz}$ and $3.5 \mathrm{GHz}$ gain of between $2 \mathrm{~dB}$ to 4 $\mathrm{dB}$, can effectively receive the electromagnetic radiation. The bands below $1 \mathrm{GHz}$ antenna VSWR less than 1. 5, reducing the interference radiation itself, can be very good to accept refrigerators, televisions and other electromagnetic radiation of a weak signal.

\section{RESUlTs}

Antenna kind photo in Figure 9. Agilent N5230Atest antenna based on the amount of network analyzers its results shown in Figure10. Antennabetween0. $19 \sim 6.3 \mathrm{GHz}$ VSWR less than 2, subtle differences maybe caused by processing errors, dielectric loss or parasitics head of the media.

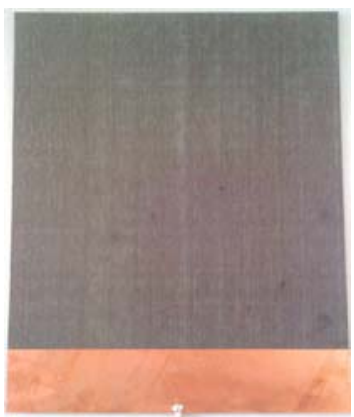

(a): Positive detection antenna

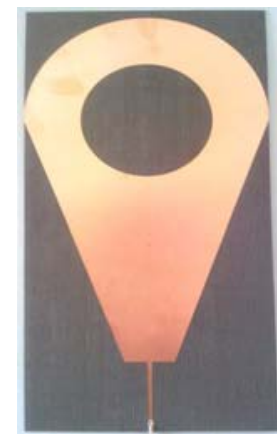

(b): The back of the antenna detection
FIGURE IX. DETECTION ANTENNA 


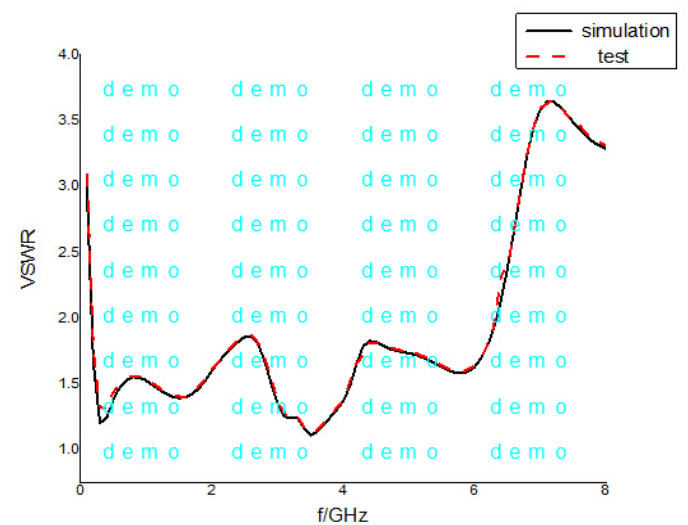

FIGURE X. ANTENNA VOLTAGE STANDING WAVE RATIO TEST AND SIMULATION COMPARISON

\section{CONCLUSION}

Electromagnetic radiation pollution directly affect the environment and human health, in order to detect the indoor electromagnetic radiation, proposed an indoor electromagnetic detection antenna, and the factors affecting the performance of the antenna are analyzed. The antenna meets the requirements of the ultra wide band antenna, by opening and increasing a paragraph of the high impedance micro-strip line . antenna's $\mathrm{S} 11 \leq-10 \mathrm{~dB}$ impedance bandwidth is $0.19 \mathrm{GHz} \sim 6.3 \mathrm{GHz}$, the relative bandwidth of 191 . 3\%. Indoor appliances can basically cover band electromagnetic radiation and antenna has good VSWR; most antenna gain between $1 \mathrm{~dB}$ to $4 \mathrm{~dB}$ in. However, further research is needed to reduce the antenna size, increase the gain.

\section{ACKNOWLEDGEMENT}

This work has been supported by National Natural Science Foundation of China (61071001), National Natural Science Foundation of China(61372094). in this, thanks for the help of Professor $\mathrm{Wu}$ and teacher Ping Lanlan.

\section{REFERENCES}

[1] LI Yan-Zhong, ZHAO Branch groom, such as the effects of electromagnetic radiation on the body's physiological indices Chinese Radiation Health . 2013 (01), 99-100.

[2] Alfonso Balmori, The incidence of electromagnetic pollution on wild mammals: A new "poison" with a slow effect on nature, The Environmental ist, 2010, 30(1)

[3] Li star, Dujun Kai, Huangchuan You , Yin Tong research 110kV high-voltage transmission lines parallel to each other when the electromagnetic environmental effects [J]. Hydroelectric Energy 2011 (06) .

[4] LIU Bao-Hua, YANG Xu-Fu , ING Feng, WANG Mei-Xia . mobile communication base station electromagnetic radiation absorbed dose characteristics and human studies [J] Mobile Communications 2010 ( 14 ).

[5] ZHONG Shun, LING Fang, YAN Xiao-Rong . Ultra-wideband planar antenna technology [J]. Radio Science, 2007, 22 ( 2 ) :308 -315.

[6] JIANG Wen , GONG Shu-xi , Hong Tao et al. Research new ultra-wideband micro-strip antenna radiation and scattering properties [J]. Radio Science 2010, 25 ( 2 ), 289 -392

[7] SHI Sheng-Jie, GUO Hui-Ping broadband antenna design and implementation of [D] Suzhou University 2009, 56-59 .

[8] Wu Yi-Jiang, HU Shao-Wen , et al . a new type of ultra- wideband antenna [J] piezoelectric and acousto-optic , 2013 (02), $201-203$.

[9] ZHONG Shun . micro-strip antenna theory and application [M], Xi'an
University of Electronic Science and Technology Press 1991, 7, 163-164.

[10] BAI XF, ZHONG SS, LIANG X L. Leaf-shaped monopole antenna with extremely wide bandwidth [J] Microwave Opt tech Lett . 2006, 48 (7).

[11] ZHANG Jun, LIU City, ZHANG Xian-Feng et al . micro-strip antenna theory and engineering [M] Defense Industry Press . 1988, 7, 294-295 